\title{
Prey capture behavior evoked by simple visual stimuli in larval zebrafish
}

\author{
Isaac H. Bianco ${ }^{1}{ }^{\text {, Adam R. Kampff }}{ }^{2}$ and Florian Engert ${ }^{1}$ \\ ${ }^{1}$ Department of Molecular and Cellular Biology, Center for Brain Science, Harvard University, Cambridge, MA, USA \\ ${ }^{2}$ Champalimaud Neuroscience Programme, Champalimaud Centre for the Unknown, Lisbon, Portugal
}

\section{Edited by:}

Federico Bermudez-Rattoni, Universidad Nacional Autónoma de México, Mexico

\section{Reviewed by:}

James W. Grau, Texas A\&M

University, USA

Stephan C. F. Neuhauss, University of

Zürich, Switzerland

*Correspondence:

Isaac H. Bianco, Department of Molecular and Cellular Biology,

Harvard University, BioLabs-2073, 16

Divinity Avenue, Cambridge, MA

02138, USA.

e-mail: ibianco@mcb.harvard.edu
Understanding how the nervous system recognizes salient stimuli in the environment and selects and executes the appropriate behavioral responses is a fundamental question in systems neuroscience. To facilitate the neuroethological study of visually guided behavior in larval zebrafish, we developed "virtual reality" assays in which precisely controlled visual cues can be presented to larvae whilst their behavior is automatically monitored using machine vision algorithms. Freely swimming larvae responded to moving stimuli in a size-dependent manner: they directed multiple low amplitude orienting turns $\left(\sim 20^{\circ}\right)$ toward small moving spots $\left(1^{\circ}\right)$ but reacted to larger spots $\left(10^{\circ}\right)$ with high-amplitude aversive turns $\left(\sim 60^{\circ}\right)$. The tracking of small spots led us to examine how larvae respond to prey during hunting routines. By analyzing movie sequences of larvae hunting paramecia, we discovered that all prey capture routines commence with eye convergence and larvae maintain their eyes in a highly converged position for the duration of the prey-tracking and capture swim phases. We adapted our virtual reality assay to deliver artificial visual cues to partially restrained larvae and found that small moving spots evoked convergent eye movements and J-turns of the tail, which are defining features of natural hunting. We propose that eye convergence represents the engagement of a predatory mode of behavior in larval fish and serves to increase the region of binocular visual space to enable stereoscopic targeting of prey.

\section{Keywords: zebrafish, prey capture, behavior, binocular vision, ocular vergence}

\section{INTRODUCTION}

The larval zebrafish is a vertebrate model that presents a number of advantages for studying the neural basis of behavior (Portugues and Engert, 2009; McLean and Fetcho, 2011). In particular, the translucency of the animal allows for non-invasive imaging of neural activity throughout the CNS (e.g., Orger et al., 2008; Muto et al., 2011) and molecular-genetic tools, including recent optogenetic methods, can be used to test the function of individual neurons within circuits (Douglass et al., 2008; Wyart et al., 2009). An important prerequisite for using zebrafish to define the circuits and computations that underlie behavior is the identification and characterization of specific zebrafish behaviors and their releasing stimuli. In addition, experimental assays must be developed in which defined stimuli can be presented to larvae to evoke particular motor outputs under conditions in which neural activity can be measured simultaneously.

From only 5 days post-fertilization, larval zebrafish start to hunt and capture live prey. Hunting behavior appears to be primarily guided by vision in larval fish and comprises a sequence of discrete locomotor maneuvers that can be deployed in variable combinations (Gahtan et al., 2005; McElligott and O'Malley, 2005). Some stereotypical behavioral elements are uniquely performed during hunting. J-turns are exclusively used during prey-tracking, and serve to orient larvae toward their prey with minimal forward displacement or hydrodynamic disturbance. The final stage of the prey capture sequence is a kinematically distinct capture swim, which culminates in biting at the prey (Borla et al., 2002).

Studies in other species provide strong evidence that the optic tectum is essential for the detection of prey and the release of appropriately targeted, species-specific, behavioral responses. Pioneering work by Ewert et al. (2001) showed that tectal neurons in toads function as "feature detectors," having sensory response properties tuned to visual stimuli with configural features characteristic of natural prey; the combined activity of such tectal neurons represents a command releasing system that elicits various action patterns comprising toad hunting behavior including orienting turns, approaches to prey, fixations and snapping. Ablations of retinal inputs to the optic tectum cause a substantial decrease in prey capture success in larval zebrafish (Gahtan et al., 2005), suggesting that as in other species, this site is an essential component of the neural circuitry that controls hunting. However, the sensorimotor computations performed by tectal circuits and the other brain regions that are downstream of the tectum, which mediate the stereotyped motor outputs that define this behavior, remain unknown.

To facilitate the study of the stimulus-response characteristics of visually guided behaviors, we first developed a "virtual world" assay for larval zebrafish in which we could present freely swimming larvae with carefully controlled visual stimuli. We found that kinematically distinct orienting and aversive responses were 
reliably evoked by moving visual cues in a size-dependant manner. Zebrafish larvae tracked small moving spots, which motivated us to examine in detail how they first respond to prey during natural hunting behavior. We found that the first behavioral element in every hunting routine was a conspicuous eye convergence response. Next, we adapted our "virtual world" paradigm to present defined visual cues to larvae that were partially restrained and therefore more experimentally accessible. In this assay, small moving spots evoked the stereotypical ocular convergence response, as well as J-bends of the tail, which define the first behavioral elements of natural hunting. Quantitative analyses of the precise sequence of eye rotations and tail movement provided insights into the sensorimotor computations underlying prey recognition. In particular, our data suggest that binocular processing of prey-like stimuli increases the probability of prey capture specific responses and the initiation of J-turns may be dependent on prior activation of convergent eye movements.

\section{RESULTS \\ "VIRTUAL WORLD" ASSAY FOR FREELY SWIMMING LARVAL ZEBRAFISH: RESPONSES TO MOVING VISUAL STIMULI}

To study visually guided behavior in larval zebrafish, we combined a $360^{\circ}$ projection method (Orger et al., 2004) with a real-time video tracking system to create a novel behavioral assay for freely swimming zebrafish larvae (Figure 1A). In this "virtual world," zebrafish (6-9 days post-fertilization, dpf) responded to different sized moving spot stimuli ( $1^{\circ}$ versus $10^{\circ}$ in diameter) with distinct behaviors (Figure 1B). Small moving spots elicited tracking-like behaviors, comprising multiple low amplitude turns in the direction of the visual stimulus, whereas large stimuli evoked highamplitude turns away from the visual cue. Although fish did not respond to every stimulus presentation (response rate: $22 \%$ for small stimuli, 24\% for large stimuli), the average change in orientation for cues of different sizes revealed a significant effect of spot size on the directionality of the behavioral responses $\left(1^{\circ}\right.$ versus $5^{\circ}, p<0.001 ; 1^{\circ}$ versus $10^{\circ}, p<0.001$, t-tests, Figure 1C). In addition to this switch in directionality, the behavioral responses to different sized cues were kinematically distinct. Considering only the first directed turn (a turn toward an attractive stimulus and away from a repulsive stimulus) following the appearance of the moving spot, we found that whilst small, attractive stimuli evoked $\sim 20^{\circ}$ turns toward the direction of stimulus motion, larger, aversive cues elicited higher amplitude $\sim 60^{\circ}$ turns in the opposite direction to the visual stimulus (Figure 1D). Larval zebrafish were also sensitive to the speed at which the spots moved. In Figure 1E, the time-course of responses to small and large spots moving at different speeds are plotted as the difference in average orientation trajectories for rightward and leftward-moving stimuli (where positive angles indicate orientation changes in the direction of the stimulus). In the case of the small spot stimulus, fish orientation trajectories have different profiles for different speeds, consistent with the idea that larvae respond to small moving cues with a visually guided tracking behavior.

\section{LARVAL ZEBRAFISH RESPOND TO PREY WITH EYE CONVERGENCE}

The orienting responses we observed in freely swimming larvae presented with small moving spots suggested that this simplified visual stimulus might be sufficient to release a component of natural hunting behavior.

To evaluate whether there are any distinctive features of the initial response of larval fish to their prey, we analyzed 118 movie sequences of $7 \mathrm{dpf}$ zebrafish hunting paramecia. We observed that during every hunting episode, larval fish converged their eyes. Convergent eye movements appeared to represent the first behavioral element in the hunting routine because they occurred at the onset of the distinctive series of prey-tracking maneuvers in which larval fish reduce the distance and angular deviation between themselves and their prey (McElligott and O'Malley, 2005). Jturns, exclusively used during prey-tracking, always occurred after, or concurrent with ocular convergence. Furthermore, the eyes were maintained at a high vergence angle throughout the hunting routine until immediately after the capture swim, when the larvae struck at the paramecia (Movie S1 in Supplementary Material).

For 39 movies, we quantified eye position at four stages during the hunting routine: (1) in the first frame immediately before the larva responded to the paramecium, (2) $62.5 \mathrm{~ms}$ (5 frames) after the onset of the initial behavioral response, defined by the onset of convergent eye movement, (3) immediately before the onset of the final capture swim, (4) $125 \mathrm{~ms}$ after the larvae struck at the paramecium (Figures 2A,B). Within $62.5 \mathrm{~ms}$ of the onset of hunting, ocular vergence angle increased substantially from $36.0^{\circ} \pm 2.23^{\circ}$ to $66.9^{\circ} \pm 1.81^{\circ}$ (mean \pm s.e.m., $p<0.01$, paired ttest), with this initial response representing $76 \%$ of the maximum vergence angle we measured. Vergence angle continued to increase slightly to $76.4^{\circ} \pm 1.44^{\circ}$ ( $p<0.01$, paired t-test $)$ just before the capture swim. Within $125 \mathrm{~ms}$ of biting at the paramecium, vergence angle declined sharply to $25.1^{\circ} \pm 3.52^{\circ}$. Thus, larvae first respond to prey with convergent eye movements and a high vergence angle is maintained for the duration of the hunting routine.

Eye convergence was initiated from a range of starting eye positions and moved the eyes to a symmetrical, highly converged orientation (Figure 2C). Comparing the angular position of the left and right eyes with respect to the anterior-posterior midline of the head showed that convergence resulted in a more symmetrical eye orientation (difference in absolute angle of the left and right eyes before eye convergence $11.8^{\circ} \pm 1.54^{\circ}$, after convergence $\left.6.5^{\circ} \pm 0.86^{\circ}, p=0.013\right)$. Accordingly, the extent of eye rotation during convergence was linearly related to the initial position of the eye (Figure 2D). Thus, if the eye was initially at a temporal position in the orbit, there was a large, nasally directed rotation, whereas if the eye started in a nasal position, the magnitude of eye movement was reduced.

In $55 \%(n=64 / 116)$ of trials, the initial response of zebrafish to the paramecium included a J-turn, in addition to the ocular convergence response. J-turns are defined by two or more unilateral J-bends, which have high bend amplitude $\left(>90^{\circ}\right)$ and a caudal bend location, close to the end of the tail (McElligott and O'Malley, 2005). We analyzed the morphology of the tail at the peak of the first J-bend and found that $71.9 \pm 1.4 \%$ of the cumulative angle of the tail was localized to the final $\sim 20 \%$ of tail length (Figure 2E). Model tails reconstructed from average tail angle profiles showed the distinctive "J"-shaped morphology characteristic of this orienting maneuver (Figure 2F). For trials in which larvae performed J-turns concurrent with the 
A

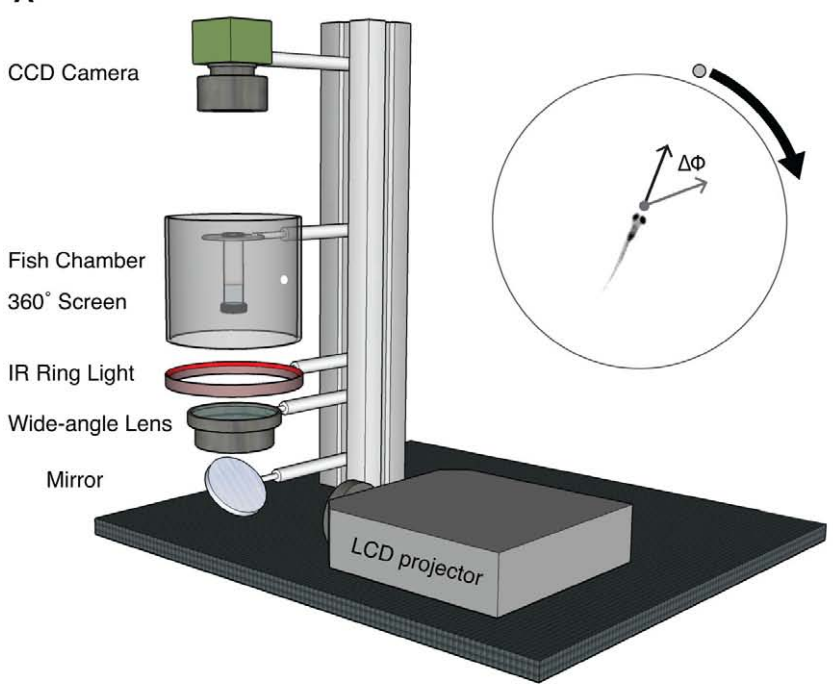

B

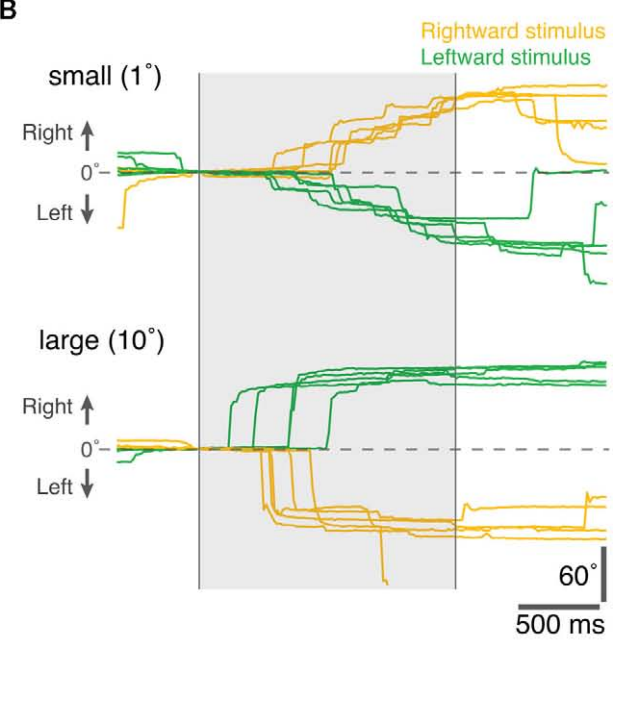

E

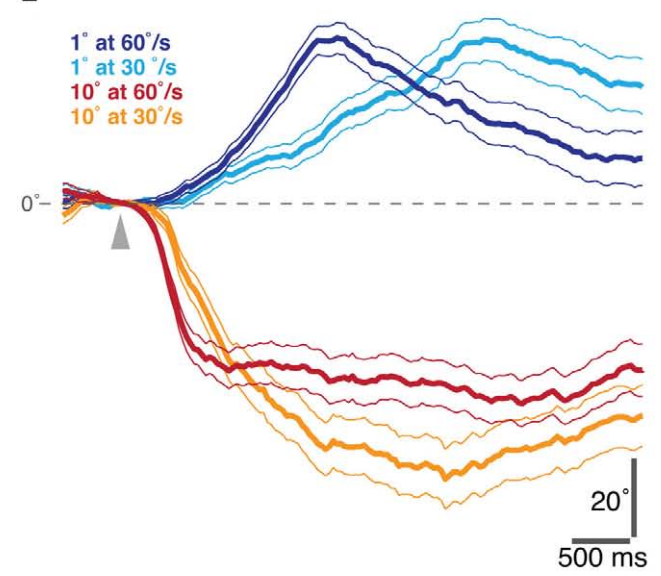

C

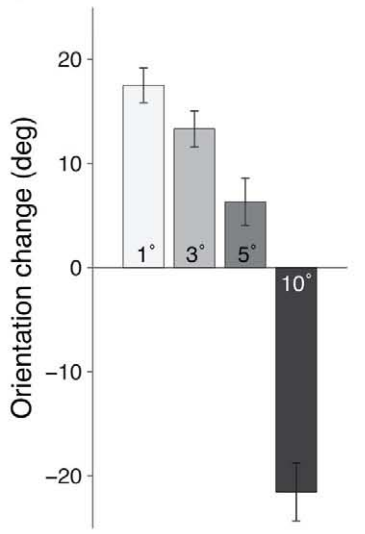

D

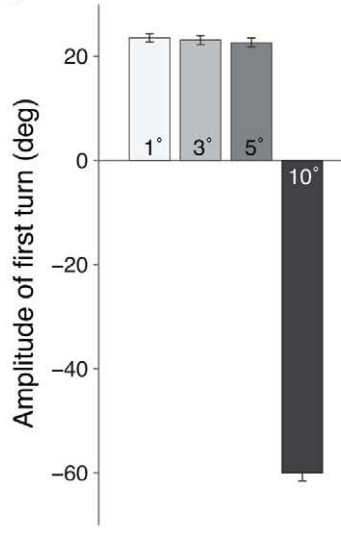

FIGURE 1 | Behavioral responses of freely swimming zebrafish to moving visual stimuli presented in a "Virtual World." (A) Schematic diagram of the "virtual visual world" assay for freely swimming larval zebrafish. An LCD projector presents the computer-generated stimulus, via a wide-angle lens, onto a $360^{\circ}$ screen. The fish is suspended within a transparent chamber held at the center of the screen and illuminated with an infrared ring-light from below. An infrared-sensitive CCD camera continuously tracks the position and orientation of the swimming zebrafish in real-time $(40 \mathrm{~Hz})$. At the start of each trial, the visual stimulus - a moving circular spot of varying size and speed - was positioned directly in front of the fish and then moved $90^{\circ}$ to either the left or right, whilst the change in orientation of the fish $(\Delta \phi)$ was recorded in real-time (inset). (B) Example orientation trajectories for fish presented with rightward (yellow) and leftward (green) moving small (top: $1^{\circ}$ diameter, $60^{\circ} / \mathrm{s}$ ) and large (bottom: $10^{\circ}$ diameter, $60 \%$ s) visual stimuli. The shaded region indicates the period of stimulus presentation. Positive angles indicate a rightward change in the orientation of the larva, and negative angles a leftward change. (C) The mean change in orientation during the presentation of moving spots of different sizes, error bars report s.e.m. $\left(1^{\circ}, n=705 ; 3^{\circ}, n=690 ; 5^{\circ}, n=646\right.$; $\left.10^{\circ}, n=615\right)$. Positive orientation changes are in the direction of stimulus motion. (D) The mean amplitude of the first directed turn, i.e., directed toward the attractive stimuli $\left(1^{\circ}, 3^{\circ}\right.$, and $5^{\circ}$ diameter spots $)$ and directed away from the repulsive stimulus ( $10^{\circ}$ diameter spot), error bars report s.e.m. $\left(1^{\circ}, n=366 ; 3^{\circ}, n=343 ; 5^{\circ}, n=327 ; 10^{\circ}, n=330\right)$. (E) The difference between the average orientation trajectories for stimuli moving leftward versus rightward are shown for an attractive $\left(1^{\circ}\right)$ and repulsive $\left(10^{\circ}\right)$ spot moving at two different speeds (fast, $60 \%$ s and slow, $30 \%$ s). The arrowhead marks the time at which the stimulus appears. The different time-courses of the response trajectories for the small (attractive) stimuli of different speeds suggests a visual tracking behavior, possibly related to the tracking phase of natural prey capture; error lines report s.e.m. $\left(1^{\circ}\right.$ at $60^{\circ} / \mathrm{s}, n=705 ; 1^{\circ}$ at $30^{\circ} / \mathrm{s}$, $n=561 ; 10^{\circ}$ at $60^{\circ} / \mathrm{s}, n=615 ; 10^{\circ}$ at $30^{\circ} / \mathrm{s}, n=479$ ). initial vergence eye movements, careful frame-by-frame analysis of movie sequences showed that in $65 \%$ of cases the ocular and tail responses appeared to initiate concurrently (in the same video frame), whereas in the remaining trials J-turns clearly began after the onset of convergence movements (range 1-3 video frames at $80 \mathrm{~Hz}$ ). However, in no case did the tail begin to move before the eyes. Therefore, the ocular convergence response can be considered to be the first motor output in the hunting sequence. 
A

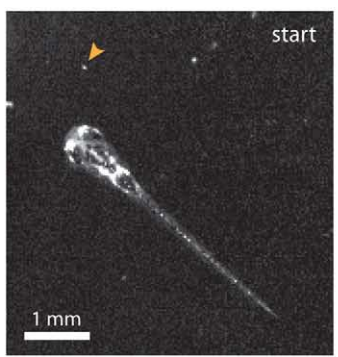

B

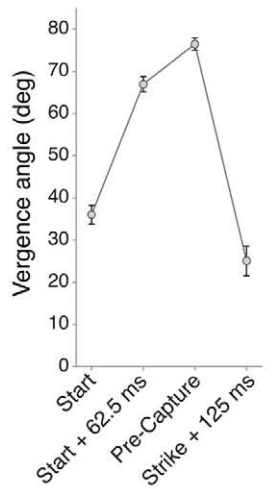

$\mathbf{E}$

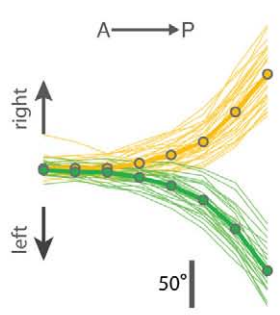

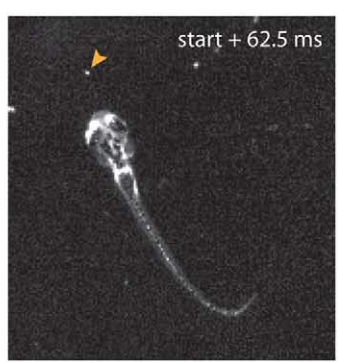

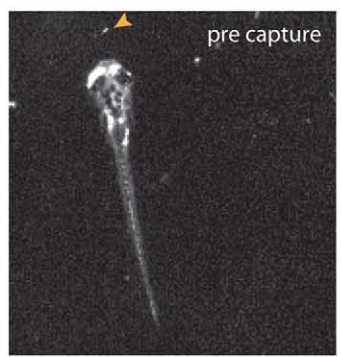

C

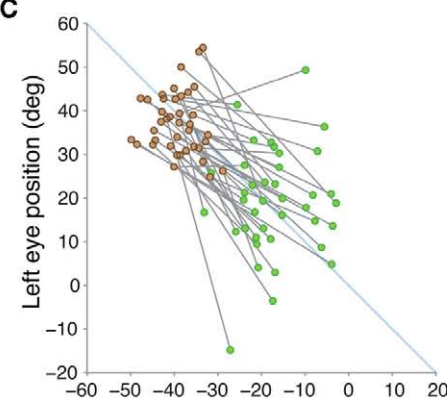

Right eye position (deg)

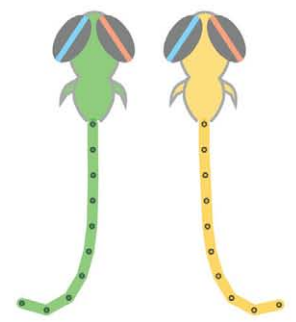

H all responses

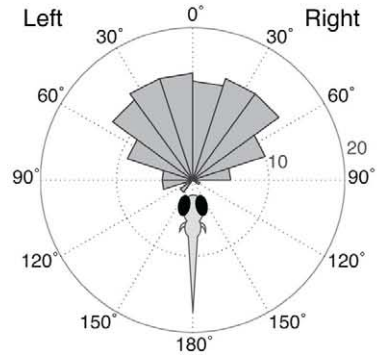

FIGURE 2 | Zebrafish larvae respond to paramecia with eye convergence during hunting. (A) Individual frames from a movie sequence of a typical hunting episode showing the 4 stages at which we quantified eye position. The paramecium is indicated by an orange arrowhead. In this case, a J-turn occurs concurrently with eye convergence when the larva first responds to the paramecium. (B) Eye vergence angle (mean \pm s.e.m.) at four stages in the hunting routine. (C) Change in right and left eye positions from the start of the hunting episode (green symbols) to the pre-capture frame (brown symbols). Note that nasal rotations correspond to a numerical increase in left eye position and a numerical decrease in right eye position. (D) Change in eye position (start $\rightarrow$ pre-capture) versus starting eye position for the left (blue) and right (red) eyes. Note that right eye positions were inverted (multiplied by -1 ) in this plot to aid comparison between the eyes. (E) Cumulative tail angle
D

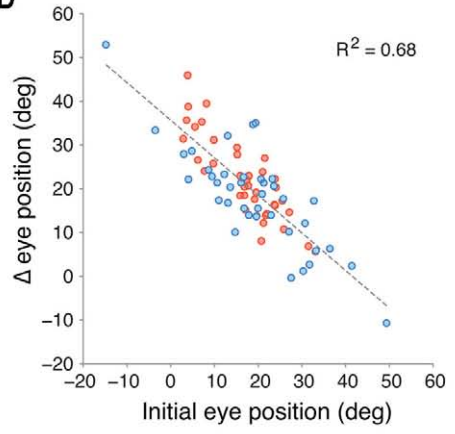

G

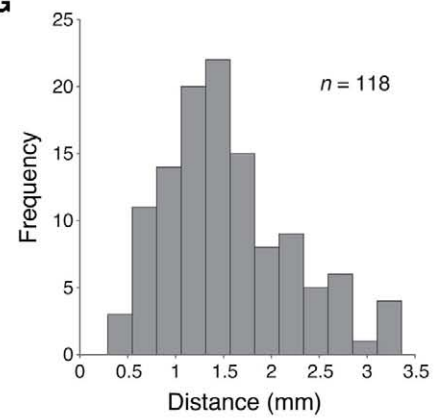

Initial eye position (deg)

Distance $(\mathrm{mm})$
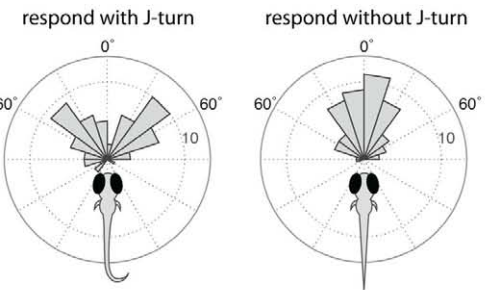

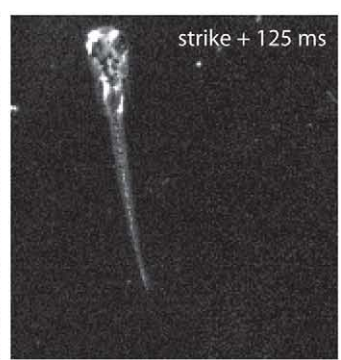

at the peak of the first J-bend in 62 trials (33 right (yellow) and 29 left (green) J-bends) where larvae first responded to the paramecium with a J-turn in addition to eye convergence. Cumulative angle is plotted as 8 values from the anterior to posterior end of the tail. Thick lines with symbols show mean tail angle profiles. (F) Schematics showing model tails reconstructed from the mean tail angle profiles in (B). The eyes are also depicted at the mean vergence angle $62.5 \mathrm{~ms}$ after larvae respond to their prey. (G) Distribution of distances between the center of the fish's head and the paramecium when the larva first responds to prey. $\mathbf{( H )}$ Distribution of angular locations of the paramecia, measured from the extended midsaggital axis of the fish, when the larva first responds to prey. Left panel shows data for all responses and center and right panels show data segregated according to whether the first orienting response included a J-turn or not. 
To gain insights into the characteristics of the sensory stimulus that evokes hunting behavior, we measured the distance and angular location of the paramecium relative to the center of the fish's head when the larva first responded to its prey (defined by the first frame preceding the onset of eye convergence). Zebrafish responded to paramecia located at an average distance of $1.55 \pm 0.66 \mathrm{~mm}$ (Figure $2 \mathrm{G}$ ), within an arc extending $\sim 60^{\circ}$ into the left and right visual hemifields (Figure $\mathbf{2 H}$ ). When the initial response included a J-turn in combination with eye convergence, paramecia were located at more eccentric angular locations than when J-turns did not form part of the initial response (Figure $\mathbf{2} \mathbf{H}$, insets).

In summary, convergent eye movements define the onset of the larval zebrafish hunting routine. This ocular response represents the first behavioral element by which larvae react to their prey and a high vergence angle is maintained throughout the prey-tracking and capture swim phases.

\section{ARTIFICIAL VISUAL STIMULI EVOKE PREY CAPTURE ASSOCIATED RESPONSES IN RESTRAINED LARVAE}

We adapted our "virtual world" assay to allow precisely defined visual stimuli to be presented to larval zebrafish under conditions in which they were restrained and therefore more amenable to physiological analyses of neural activity patterns.

Zebrafish larvae, partially restrained in agarose but with their eyes and tail free to move, were presented with moving visual spots projected onto a semicircular screen. A high-speed camera and machine vision algorithms were used to extract eye and tail movements during the presentation of different visual cues (Figures 3A,B).

Small moving spots, which appeared directly in front of the fish and then swept to the left or right sides (angular velocity $30 \%$ ), evoked highly stereotyped responses. Qualitatively, the evoked eye and tail movements closely resembled the natural behavior of larvae during hunting routines, when they first respond to paramecia. Figure 3C shows example trials from the same zebrafish responding to a leftward-moving spot and a rightward-moving spot. In both cases, presentation of the visual stimulus evoked eye convergence and unilateral tail bending toward the direction in which the spot was moving. The tail assumes the same characteristic "J" shape observed when freely behaving larvae perform J-turns to orient toward paramecia during prey-tracking. Movies S2 and S3 in Supplementary Material show two additional examples from another larva responding to a leftward and a rightward-moving spot.

We recorded 36 responses to 270 presentations of leftwardmoving spots and 11 responses to 251 presentations of rightwardmoving spots, in 21 fish. Eye convergence also occurred spontaneously, but at a lower frequency than during visual stimulus presentation (response rate for trials in which no stimulus was presented, $0.0022 \mathrm{~Hz}$; for leftward-moving spot presentations, $0.0478 \mathrm{~Hz}, p<0.001$; for rightward-moving spots $0.0241 \mathrm{~Hz}$, $p=0.07$; paired t-tests).

\section{QUANTIFICATION OF OCULAR AND TAIL RESPONSES}

We quantified features of the eye and tail responses of restrained larvae that were evoked by moving visual spots, both to evaluate the extent to which these behaviors resemble natural prey capture associated responses and to gain insights into underlying neural mechanisms.

During stimulus-evoked eye convergence, mean vergence angle increased significantly from $21.1^{\circ} \pm 0.83^{\circ}$ to $55.4^{\circ} \pm 1.26^{\circ}$ $(p<0.001$, paired t-test, Figure 4A). Although vergence angles measured automatically with our machine vision algorithms were numerically lower than those measured manually for fish hunting paramecia (Figure 2B), the magnitude of convergence was indistinguishable between restrained larvae responding to moving spots $\left(34.3^{\circ} \pm 1.33^{\circ}\right)$ compared to larvae reacting to paramecia during natural hunting $\left(30.9^{\circ} \pm 2.42^{\circ}, p=0.21\right.$, t-test $)$.

As we observed during hunting sequences, the extent of eye rotation was linearly related to starting eye position, before the larva responded to the moving spot (Figure 4B). When the eye was initially located in a nasal position, the smallest angular changes were evoked, whereas in trials where the eye started in a more temporal position, larger nasally directed ocular rotations occurred.

Interestingly, the direction of gaze, at the time the visual stimulus was presented, predicted whether or not the larvae subsequently responded to the visual cue (Figure 4C). For trials with leftward-moving spots, both eyes were initially rotated leftward at the onset of presentations that resulted in prey capture associated responses. A symmetrical result was observed for rightwardmoving spots: both eyes were rotated rightward in advance of stimulus presentations that evoked responses. In contrast, the direction of gaze showed no left-right bias for trials where the larvae did not respond. These differences in the direction of gaze for response trials versus non-response trials, were highly significant (t-tests, $p$-values in Figure 4C).

Stimulus-evoked eye convergences were associated with unilateral tail bends and the laterality of those bends was appropriately correlated with the direction of visual stimulus motion: thus leftward bends were released by leftward-moving spots and larvae responded to rightward-moving spots with rightward tail movements (Figure 4D). Although spontaneous eye convergences involved the same increase in vergence angle $\left(31.6^{\circ} \pm 0.91^{\circ}\right)$ as stimulus-evoked convergences $\left(34.3^{\circ} \pm 1.32^{\circ}, p>0.05\right.$, t-test), they were associated with less stereotyped tail movements, which displayed no overall laterality bias and had a broad range of bend amplitudes (Figure 4D).

J-turns performed by larvae during prey-tracking comprise two or more J-bends (McElligott and O'Malley, 2005) and likewise, the tail responses evoked by moving spots in restrained larvae were composed of multiple unilateral bends $(3.1 \pm 1.3$, mean \pm s.d., Figure $4 \mathrm{E}$ ). The angular profile of the tail at the peak of the first bend (indicated by a gray line in Figure 4E) showed a characteristic caudal location in maximum bend amplitude (Figure $4 \mathbf{F}$ ), wherein $63.1 \pm 7.6 \%$ of tail curvature was localized to the most caudal $\sim 20 \%$ tail length, similar to the J-bends we measured for freely hunting larvae (compare with Figure $2 \mathrm{E}, p=0.22$, t-test comparing proportion of tail curvature contributed by final two tail segments for larvae hunting paramecia versus larvae responding to moving spots). Model tails reconstructed from the average tail angular profiles (Figure 4G) show the far-caudal bending characteristic of prey-tracking J-turns. These reconstructed tails are not 
A
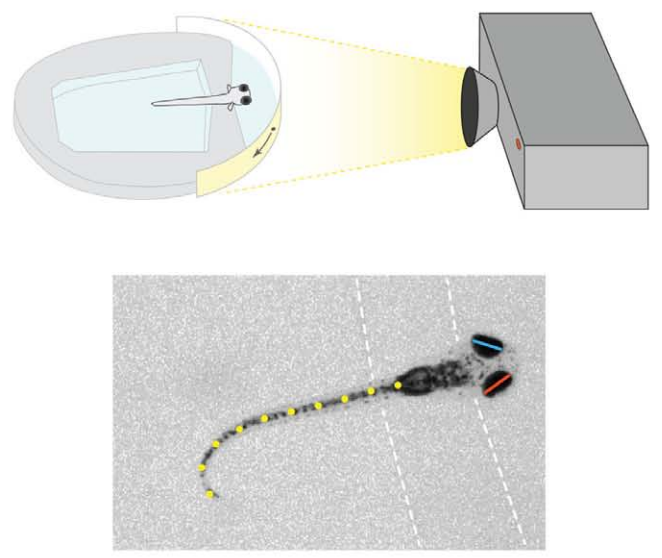

C
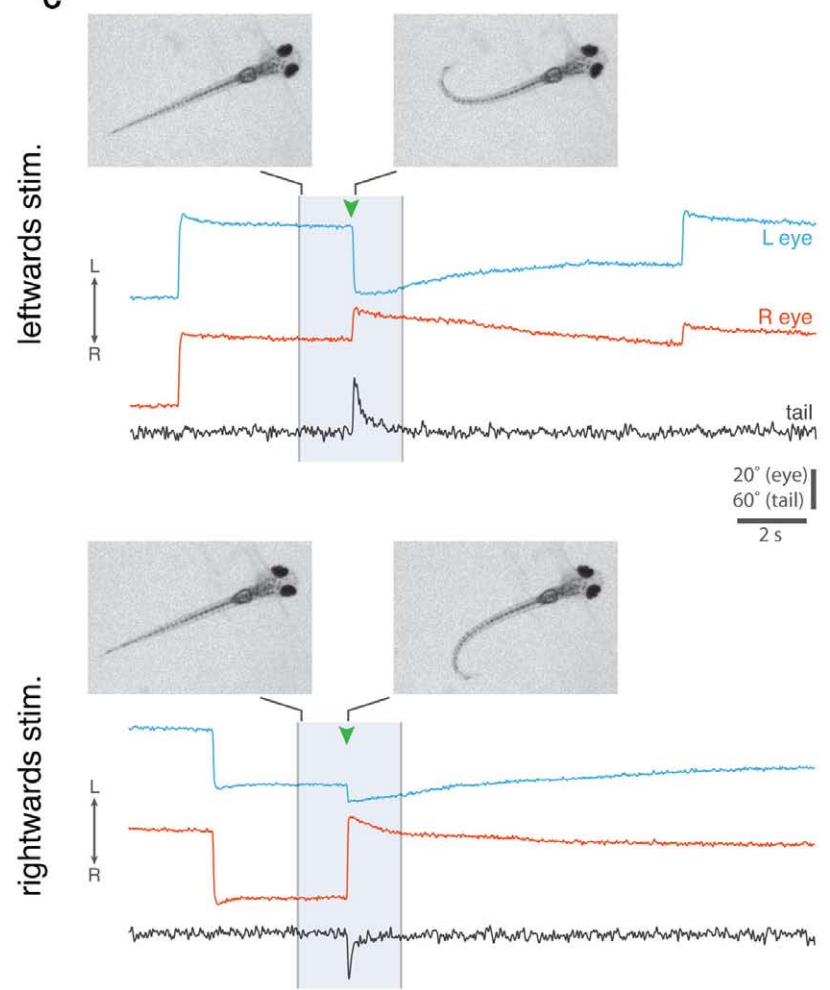

B

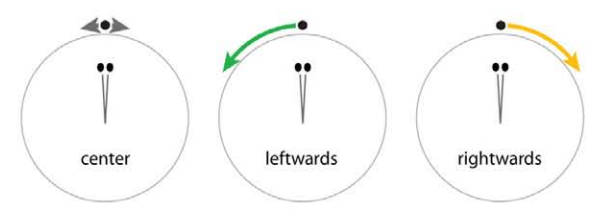

D

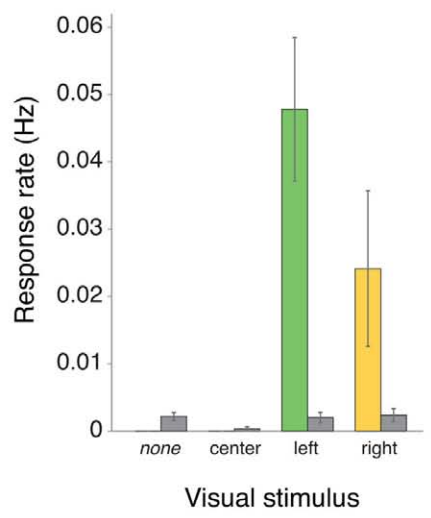

FIGURE 3 | Prey capture assay for partially restrained larval zebrafish.

(A) Top, Schematic of the set-up for presenting visual cues to larvae that are partially restrained, but free to move both their eyes and tail. Visual stimuli are projected onto a semicircular diffusive screen in front of the fish and eye and tail movements are recorded from above with a high-speed camera, under infrared illumination (not shown). Bottom, Example camera frame showing automated machine vision tracking of left (blue) and right (red) horizontal eye position, and tail morphology (defined by $10 x-y$ co-ordinates, shown in yellow). Dashed lines indicate the boundaries of the agarose. (B) Schematic of three visual stimuli, which we presented to larval fish. Small white spots either oscillated back and forth directly in front of the larva for $5 \mathrm{~s}$ ("center"), or appeared directly in front of the fish and moved $\sim 90^{\circ}$ to the left ("leftward") or right ("rightward") in 3s. (C) Examples of two complete $20 \mathrm{~s}$ trials from the same larva, presented with a leftward-moving spot (top) or rightward-moving spot (bottom). The $3 \mathrm{~s}$ visual stimulus presentation is indicated by the shaded region. At the times indicated by green arrowheads, the larva responded with nasally directed rotations of both eyes (i.e., convergence eye movements) and unilateral tail bending toward the direction of visual stimulus motion. Images show single video frames at the onset of stimulus presentation and at the peak of the first J-bend of the tail. Notice that larvae also perform spontaneous conjugate saccades, which are visible in these traces in the periods surrounding stimulus presentation, and that the eyes are rotated leftward in advance of the larva responding to the leftward-moving spot and rightward in advance of the response to the rightward-moving spot. Note that data traces have been reflected about the $x$-axis for presentation, such that eye convergence is represented by a decrease in the distance between the left and right eye position traces (see Methods). (D) Frequency of eye convergence responses for different visual stimuli (mean \pm s.e.m., $n=21$ fish). Colored bars indicate responses during the $3 \mathrm{~s}$ stimulus presentation, gray bars indicate spontaneous responses that occurred in the remaining portions of the $20 \mathrm{~s}$ trial when no stimulus was shown (for "none" trials, no stimulus was shown during the entire $20 \mathrm{~s}$ trial). 
A

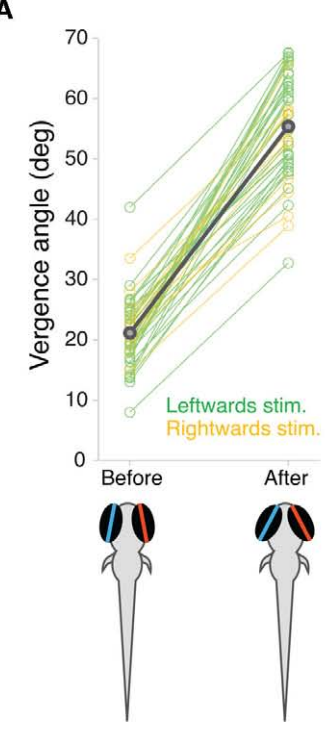

B
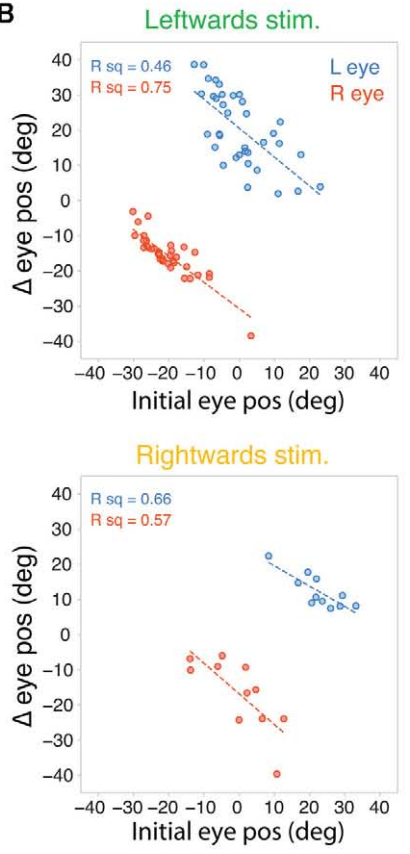

C

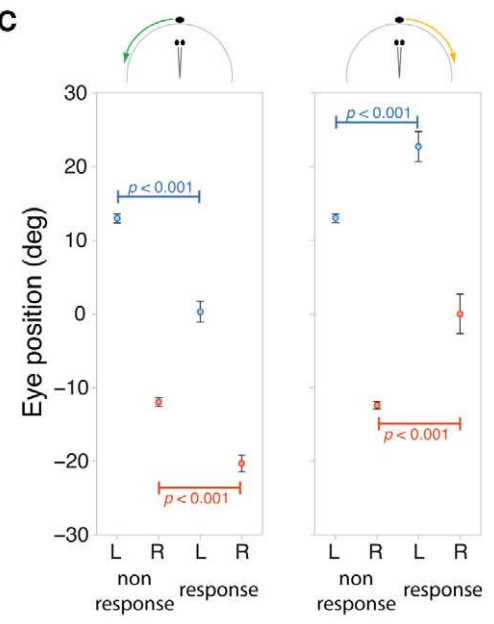

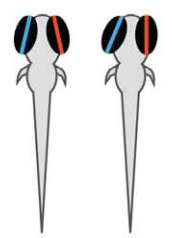

D

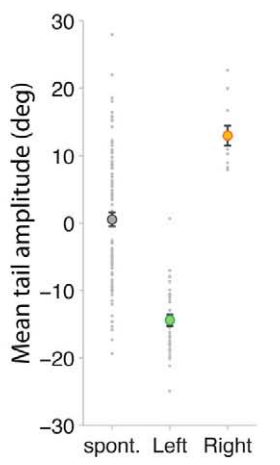

E

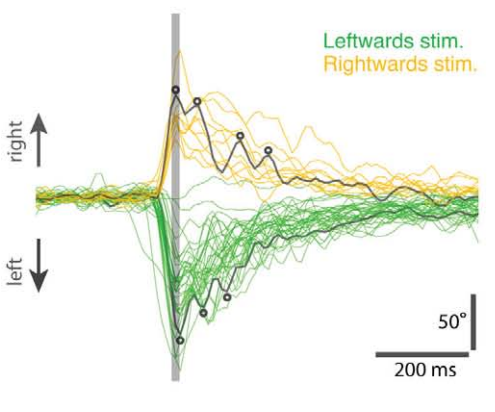

$\mathbf{F}$

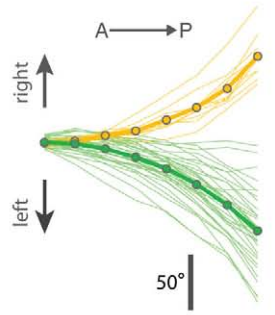

G

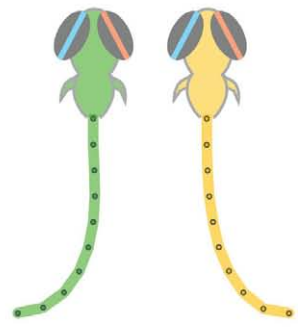

FIGURE 4 | Quantification of eye and tail responses. (A) Eye vergence angle before and after convergence responses to leftward (green) and rightward (yellow) moving stimuli. Thick gray bar shows mean vergence angles which are also depicted in the schematics. (B) Change in eye position during eye convergence plotted against initial eye position for the left (blue) and right (red) eyes for trials with leftward (top) and rightward (bottom) moving spots. (C) Initial eye position, before visual stimulus presentation, for trials in which the fish responded to the stimulus versus trials in which there was no response. Data shown as mean \pm s.e.m. and mean eye positions are depicted in schematics below the plots. (D) Mean cumulative angle of the tail measured in a $1.2 \mathrm{~s}$ time window surrounding spontaneous and stimulus-evoked eye convergence responses. Circles indicate average values across all responses (mean \pm s.e.m.). (E) Tail responses associated with eye convergence for leftward (green) and rightward (yellow) moving spot trials. Total cumulative tail angle is plotted against time and traces are aligned to the peak of the first tail bend, indicated by the vertical gray line. Two examples have been colored dark gray to highlight the multiple unilateral tail bends (indicated by small circles), which characterize the responses. (F) Cumulative tail angle along the length of the tail (anterior $\rightarrow$ posterior) at the peak of the first J-bend (time marked by vertical gray line in (E)). Thick lines with symbols show mean profiles. (G) Schematics showing model tails reconstructed from the mean tail angle profiles in (F). The eyes are shown at the mean vergence angle following the eye convergence response. quite as "J" shaped as those measured for larvae hunting paramecia because our machine vision algorithm did not track all the way to the end of the tail during high-speed acquisition, and so the full extent of tail curvature was likely underestimated.

In summary, both ocular and tail responses evoked by moving spots closely resemble the initial responses of freely swimming larvae reacting to moving prey, providing strong support that our assay successfully enables prey capture related behaviors to be driven by simplified visual cues in restrained larval zebrafish.

\section{TIMING OF EYE AND TAIL RESPONSES}

We evaluated the timing sequence of the eye and tail responses as well as the angular location of the moving visual spot at the instant the larvae first responded to it. 
When larvae responded to leftward-moving spots, nasal rotation of the right eye was consistently the first motor output comprising the response (Figures 5A,B). The onset of convergent movement of the right eye preceded tail motion by $20 \mathrm{~ms}$, $(p<0.001$, Wilcoxin sign rank test) and left eye rotation by $30 \mathrm{~ms}(p<0.001$; Figure 5B). There was no consistent temporal sequence between left eye rotation and tail movement $(p>0.05)$ : in some cases the left eye started moving before the tail and in other cases after the tail. The reverse was true for responses to rightward-moving spots, where movement of the left eye was the first component of the response, occurring $20 \mathrm{~ms}$ before tail movement $(p=0.004)$.

Interestingly, in approximately one quarter of responses, both eyes initially displayed a very brief conjugate rotation in the direction of the moving spot and then one eye reversed direction to produce eye convergence. Thus, in 9/36 responses to leftwardmoving spots, both eyes initially tracked toward the left and then the left eye reversed direction and rotated nasally (green arrow in example trace in Figure 5A). Similarly, in 3/11 trials where larvae responded to rightward-moving spots, both eyes briefly rotated conjugately toward the right, before the right eye changed direction from temporal to nasal to produce eye convergence (Figure 5A). This finding suggests that larval fish may first respond to small moving spots with conjugate eye movements but this response is rapidly replaced by disjunctive rotations producing eye convergence.

In our assay, moving spots appeared directly in front of the larvae and moved in a nasal-temporal direction in either the left

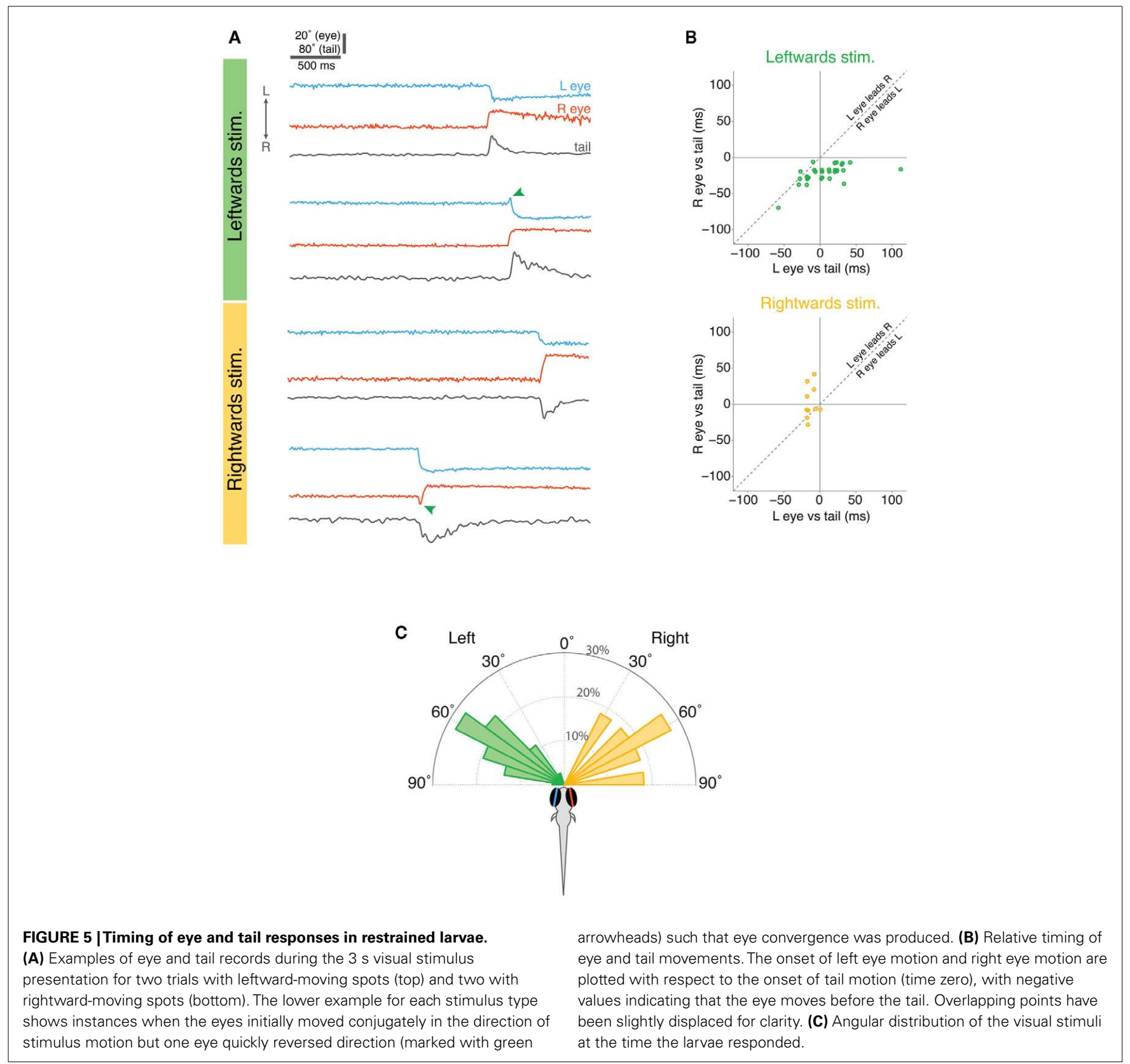


or right visual hemifield. The majority of behavioral responses occurred when the spots reached approximately $60^{\circ}$ from the extended midsaggital plane of the fish $(\sim 1.9 \mathrm{~s}$ into the $3 \mathrm{~s}$ stimulus presentation) (Figure 5C). Thus, the distribution of locations of the spot at the time larvae reacted to it, overlaps with the angular distribution of paramecia that larvae respond to during hunting.

\section{DISCUSSION}

We have developed assays for both freely swimming and restrained larval zebrafish in which visually guided goal-directed behaviors can be automatically and quantitatively measured. In a virtual visual world, freely swimming larvae showed distinctive orienting and aversive turns in response to moving visual cues, and a single parameter of the stimulus, its size, was sufficient for controlling selection of the behavioral response. Analyses of zebrafish hunting paramecia uncovered a novel oculomotor behavior, eye convergence, which comprises the first response of larvae to their prey. We adapted our virtual world assay for restrained larvae and used simplified visual stimuli to evoke the eye convergence and J-turn responses that define the first stage in the elaborate prey capture routine.

Freely swimming larvae oriented toward small moving spots, but as the size of the visual cue increased their behavior switched to an aversive turn. Classic studies in toads yielded similar observations: whilst toads orient toward small moving squares, as the size of the stimulus increases the toads firstly become unresponsive and then as size increases further the toads show a distinct avoidance behavior (Ewert et al., 2001). This was interpreted as a switch from a prey capture associated orienting response to an adaptive predator avoidance response. The behaviors we observed in larval zebrafish differed not only in directionality but also in terms of their kinematics. Orienting turns caused smaller changes in heading angle and larvae usually performed multiple turns in succession to track the moving stimulus. This is reminiscent of the successive J-turns larvae use to track paramecia, each of which changes heading angle by $10^{\circ}-25^{\circ}$ (McElligott and O'Malley, 2005). In contrast, avoidance turns produced large changes in heading direction $\left(\sim 60^{\circ}\right)$ and larvae usually responded to the stimulus with only one such turn. High-amplitude " $\mathrm{C}$ bends" are a defining feature of the startle response of larval zebrafish to tactile, acoustic or vibrational stimuli (two distinct types of C-bend have amplitudes of $75^{\circ}$ or $105^{\circ}$, Kimmel et al., 1974; Liu and Fetcho, 1999; Burgess and Granato, 2007) and larval zebrafish move away from large spots that oscillate back and forth below part of their swim chamber ("bouncing ball" stimuli), which has been interpreted as avoidance of a threatening, predator-like stimulus (Creton, 2009; Pelkowski et al., 2011). Thus, the high-amplitude turns we observed, which are explicitly directed away from large moving visual cues, likely constitute the motor output mediating visually guided predator avoidance.

The observation that small moving spots evoked multiple orienting turns suggested that this simple visual cue was sufficient to release components of natural hunting behavior. We examined in detail how larvae first respond to their prey and found that eye convergence not only defined the initial response, but a high vergence angle persisted for the entire duration of hunting episodes. Zebrafish are known to perform a robust optokinetic reflex (OKR) and vestibulo-ocular reflex (VOR), which contribute to gaze stabilization, as well as spontaneous conjugate saccades (Easter and Nicola, 1997; Beck et al., 2004). However, convergent eye movements of the type we observed, have not been reported in any species of larval fish (e.g., Hunter, 1972; Munk, 1995; MacKenzie and Kiorboe, 2000; Vollset et al., 2011). Notably, electrical microstimulation of the extreme anteromedial tectum evokes convergent saccades in adult goldfish, and it was suggested that this is important for food-catching behavior (Salas et al., 1997).

During eye convergence, both eyes rotated nasally and vergence angle approximately doubled. Final eye position was highly stereotyped, with the eyes symmetrically oriented with respect to the anterior-posterior axis. Due to variations in initial eye position, the left and right eyes often rotated by quite different amounts. There was a linear relationship between starting eye position and the extent of rotation such that when the eye was initially in a more temporal orientation, the magnitude of nasal rotation was greater. It is likely that convergence movements rotate both eyes to the nasal limit of the oculomotor range independent of the starting position of the eye, as has been suggested for the convergent saccades evoked by tectal microstimulation in goldfish (Salas et al., 1997).

This observation suggests that the function of eye convergence during hunting behavior in larval zebrafish is not to direct gaze toward the prey, because the eyes adopted a symmetrical converged configuration independent of prey location. Rather, it is more likely that eye convergence acts to increase the binocular overlap between the visual fields of the left and right eyes in preparation for prey-tracking. We can obtain a rough estimate of the scale of the binocular visual field (in the horizontal plane) if we assume the distance between the optical centers of the left and right eyes is $458 \mu \mathrm{m}$ and the extent of the retinal field (the region of the outside world imaged onto the retina) is $163^{\circ}$ (for $4 \mathrm{dpf}$ larvae, Easter and Nicola, 1996; see diagrams in Figure 6A). Mean vergence angle was $36^{\circ}$ in free-swimming larvae before they reacted to paramecia, predicting that visual space $>1.4 \mathrm{~mm}$ in front of the larvae would fall within the visual field of both eyes. Just prior to the capture swim, mean vergence angle increased to $76.4^{\circ}$, suggesting the region of binocular overlap advances to as close as $400 \mu \mathrm{m}$ in front of the fish (Figure 6A). This corresponds to the binocular visual field increasing from 12 to $36 \%$ of visual space.

Larval zebrafish hunted paramecia located within an arc extending $\sim 60^{\circ}$ either side of their extended midsaggital plane, which agrees well with the angular extent of the reactive perceptive field of anchovy larvae (Hunter, 1972). J-turns occurred concurrently with convergence when larvae responded to paramecia located at more eccentric angular locations. During prey-tracking, the eyes maintained a converged orientation and, as described by McElligott and O'Malley (2005), larvae using variable combinations of slow swims and J-turns to reduced the distance and angular deviation between themselves and their prey. Thus, during hunting episodes, swimming and turning maneuvers serve to change the direction of gaze, such that prey items are brought to the extended midsaggital plane of the fish as it approaches its prey. The final stage in the hunting routine is a kinematically distinct 


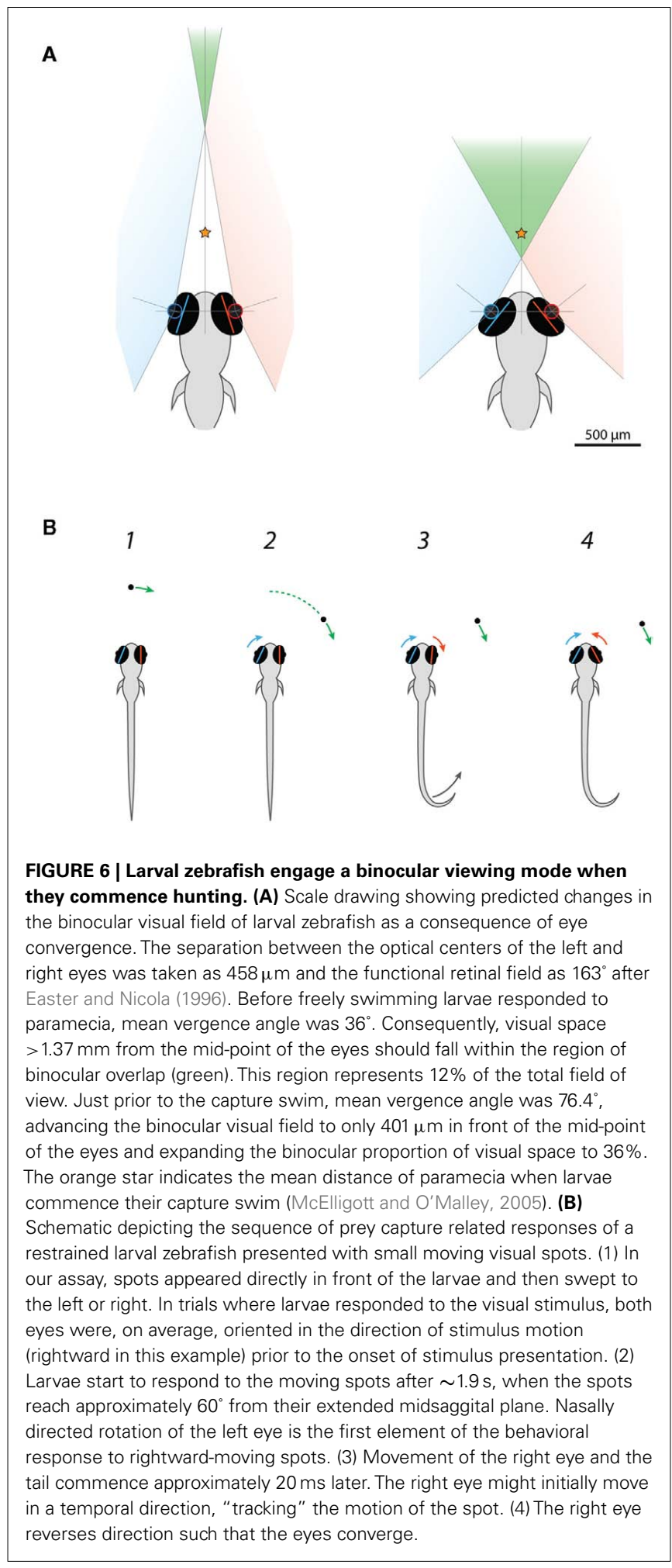

capture swim, during which larvae dart forward and open their mouths to bite at prey. This stereotyped motor output is released when prey are located directly in front of the larvae at a distance of $0.59 \pm 1.63 \mathrm{~mm}$ (mean \pm s.d., McElligott and O'Malley, 2005). In several cases we observed larvae making fine adjustments to their final position prior to the capture swim, including cases where they used pectoral fin movements to "back-up" and slightly increase the distance to their prey. Similarly, anchovy larvae use fin movements to make small adjustments to prey distance whilst they are in an S-bend configuration just prior to striking (Hunter, 1972). We propose that eye convergence allows larval zebrafish to use binocular vision to position themselves precisely with respect to their prey. This hypothesis is supported by our prediction that eye convergence advances the region of binocular visual space to less than $0.5 \mathrm{~mm}$ in front of the fish, thus enabling stereoscopic viewing of the region in which paramecia are positioned at the time capture swims are initiated. Thus, larvae could potentially judge prey distance using binocular horizontal disparity information. Notably, because the disjunctive vergence eye movements place the eyes in a stereotypical, symmetrical orientation, it is possible that a very simple binocular triangulation mechanism, such as has been proposed for preying mantids (see Prete et al., 1999), could be used for detecting that prey is appropriately located. Specifically, corresponding "trigger zones" in the left and right retinae (or in a downstream visual processing center) could signal when a small prey object was located both directly in front of the head and at a fixed, appropriate striking distance. Such a mechanism would not require information about current eye position or explicit computation of prey distance. However, whilst this simple neural mechanism might be sufficient for the final assessment of prey location and the release of the capture swim, during prey-tracking larvae need to determine the distance and angular location of prey at a variety of locations, suggesting they use more flexible binocular or monocular mechanisms to direct J-turns and slow swim maneuvers.

We developed a "prey capture" assay for partially restrained larvae in which we could evoke specific, ethologically relevant, components of hunting behavior by presenting fish with simplified visual stimuli. Larvae responded to small moving spots with prey capture specific eye and tail movements. The magnitude of convergent eye movements was the same as we observed in freely hunting larvae. Also, as observed during natural hunting, the extent of disjunctive eye rotations was linearly dependant on initial eye position and resulted in the eyes assuming a symmetrical converged orientation with respect to the anterior-posterior axis. The majority of ocular responses were accompanied by multiple, unilateral tail bends, which were indistinguishable from the J-bends that comprise J-turns used by freely swimming larvae to reorient toward prey. Restrained larvae responded to moving spots once they reached $\sim 60^{\circ}$ from the midsaggital axis; therefore, the occurrence of J-turns matches the behavior of hunting larvae, which also responded to paramecia located at these angular locations with Jturns. It is not clear why restrained larvae did not respond to moving spots at less eccentric angular locations, in contrast to the broader reactive field of freely swimming larvae during hunting.

Interestingly, we noticed that the direction of gaze, at the time moving spots were presented, predicted whether or not the larvae responded to the visual cue. Both eyes were rotated toward the right in advance of trials where rightward-moving spots evoked responses and vice versa for leftward-moving spots. Furthermore, nasal rotation of the left eye was the initiating event in responses to rightward-moving spots, and movement of the right eye was 
the first motor output in the response to leftward-moving spots (see schematic behavioral sequence in Figure 6B). These observations suggest that processing of the visual stimulus by the eye system contralateral to the direction of motion is important for releasing a prey capture specific response. Larvae were positioned $7 \mathrm{~mm}$ from the diffusive screen and so the stimulus would initially be located within the binocular region of visual space when it first appeared (Figure 6A). When larvae are looking toward the right, this binocular region is also shifted toward the right side of the midsaggital plane. Thus, the relationship between responsiveness and direction of gaze suggests larvae are more likely to respond to the cue when it is visible to both eyes for a greater period of time. However, behavioral responses (including the initial nasal rotation of the contralateral eye) did not commence until the spots reached $\sim 60^{\circ}$, which is well outside the binocular visual field. Therefore, if processing of the cue by the contralateral eye system is important for controlling the response, there must be a short term memory mechanism that allows it to persist once the cue leaves the monocular visual field of the contralateral eye.

In several trials, we observed an initial conjugate eye rotation in the direction of stimulus motion, which was rapidly followed, within milliseconds, by reversal in rotation of the ipsilateral eye to produce eye convergence. Thus, in some cases larvae appeared to initiate a conjugate saccade that would serve to redirect gaze toward the stimulus, but this motor program was quickly overridden by the eye convergence response. Interestingly, as discussed above, eye convergence does not redirect gaze toward prey, but rather moves the eyes into a stereotyped, forward-facing configuration. We propose that this novel oculomotor response represents the engagement of a predatory mode of behavior at the onset of hunting and functions to increase the extent and proximity of binocular visual space directly in front of the fish to allow accurate localization of prey.

What are the neural mechanisms that control the complex, visually guided components of prey capture behavior? By combining the assay we have developed with functional imaging of genetically encoded calcium indicators (Higashijima et al., 2003) and techniques to manipulate circuits, including optogenetics (Douglass et al., 2008; Janovjak et al., 2010; Schoonheim et al., 2010) and targeted laser-ablations (McLean et al., 2007; Satou et al., 2009; Burgess et al., 2010) it should be possible to delineate a complete circuit controlling specific components of prey capture behavior in a behaving animal. Tectal circuits are likely to be central to the sensorimotor transformations which detect prey-like visual stimuli and initiate the motor outputs that comprise the hunting sequence. Functional in vivo imaging will reveal if larval zebrafish have tectal cells similar to the T5.2 subtype described in toads, with visual responses tuned to configural features of prey (Ewert et al., 2001), and in addition provide clues as to the neural mechanism by which the selectivity of such "feature analyzing" cells is generated. Our behavioral data suggest that binocular processing of visual information is important for releasing prey capture specific motor programs; functional imaging should help to reveal where and how inputs from the two eyes are processed in the larval brain. In terms of premotor circuitry, electrical microstimulation of the rostral pole of the mesencephalic reticular formation evokes convergent saccades in goldfish (Luque et al., 2006), suggesting this region is equivalent to the dorsolateral midbrain convergence region of primates (Mays et al., 1986). Activity of premotor neurons producing eye convergence commands is expected to be a fundamental component of the activity pattern underlying all behavioral responses to prey-like stimuli in larval zebrafish.

\section{MATERIALS AND METHODS}

FISH

Zebrafish (Danio rerio) of the wild-type WIK, PE and AB strains were used for the freely swimming visual assay, wild-type $A B$ were used for all other experiments. Fish were reared on a 14/10 h light/dark cycle at $28^{\circ} \mathrm{C}$. Larvae were fed powdered nursery food at 5 and 6 days post-fertilization (dpf). Animal handling and experimental procedures were approved by the Harvard University Standing Committee on the Use of Animals in Research and Training.

\section{“VIRTUAL WORLD" ASSAY FOR FREELY SWIMMING LARVAE}

The "Virtual Visual World" assay enabled us to present visual stimuli across $360^{\circ}$ to freely swimming larval zebrafish whilst simultaneously recording behavior. The image from an LCD projector (Optoma, Japan) was reflected upward off a mirror, through a wide-angle camera lens (B\&H Photo, USA), and onto a cylindrical projection screen (100 $\mathrm{mm}$ length, $80 \mathrm{~mm}$ diameter, clear acrylic tube); the internal surface of the tube was lined with a diffusion gel (Rosco CineGel, USA) that served as a front-projection surface. An infrared-sensitive CCD camera (Hitachi, Japan) and macro-lens (Edmund Optics, USA) with a visible-light blocking filter, recorded the behavior of the fish in real-time $(40 \mathrm{~Hz})$. A custom computer-vision algorithm $(\mathrm{C}++)$ extracted the position and orientation of the fish; this information was used to position the computer-generated stimulus (DirectX, Microsoft, USA) directly in front of the zebrafish at the start of each stimulation trial. The zebrafish swam in a clear chamber constructed from a cylindrical glass cuvette $(12.5 \mathrm{~mm}$ in diameter $)$ that was partially filled with transparent Sylgard (Dow Corning, USA); the larvae swam in $\sim 4 \mathrm{~mm}$ of aquarium water, thus restricting their height to a narrow zone at the center of the arena. The fish chamber was illuminated from below with dim visible light from the projector and by a ring of infrared LEDs (Edmund Optics, USA) positioned above the wide-angle lens. The infrared light was directed at a glass diffuser affixed to the bottom of the glass cuvette and provided homogenous background illumination. Notably, the lower base of the cuvette was immersed in water contained within the small external chamber that held the diffuser. This water bath helped prevent strong reflections off the sides of the cuvette from appearing in the image recorded by the camera.

\section{HUNTING ASSAY FOR FREELY SWIMMING LARVAE}

Larval zebrafish (7 dpf) were placed in a $60 \mathrm{~mm}$ Petri dish containing aquarium water at a density of 10 larvae per dish. The dishes were obliquely illuminated using two fiber optic lights and imaged from above at $80 \mathrm{~Hz}$ using a high-speed camera (Pike F032B, Allied Vision Technologies), fitted with a machine vision lens (Kowa LM35JCM). Paramecia culture (2-4 ml, 100 parame$\mathrm{cia} / \mathrm{ml}$ ) was added to the dish and larvae were allowed to hunt the live paramecia for $1-2 \mathrm{~h}$, during which time we recorded movies 
of their hunting episodes. Complete hunting episodes, which culminated in a capture swim and biting at the paramecium, were analyzed, whether or not the larvae successfully swallowed the prey. Eye orientation was measured manually in individual video frames using Image J (NIH). Horizontal eye position was taken as the angle between the long (temporo-nasal) axis of the eye and a line drawn from the anterior end of the swim bladder through the mid-point of the snout (defining the anterior-posterior midline of the head). Positive angles are measured clockwise from the midline to the long axis of the eye and negative angles are measured counterclockwise from the midline. Thus, nasal rotation of the left eye is recorded as an increase in angular eye position and nasal rotation of the right eye as a numerical decrease in eye position (i.e., more negative). To evaluate tail morphology during the J-turns that occurred as part of the initial response of zebrafish to their prey, we analyzed a video frame of the tail at the peak of the first J-bend within the J-turn. We used the same machine vision algorithm used for the restrained larval assay (below) to extract a representation of the tail as $10 \mathrm{x}-\mathrm{y}$ co-ordinates. Cumulative tail angle was then calculated as described below.

\section{ASSAY FOR RESTRAINED LARVAE}

Larval fish were restrained by embedding them, at $6 \mathrm{dpf}$, in low melting point agarose (Invitrogen). Larvae were mounted in a 35$\mathrm{mm}$ Petri dish with their anterior-posterior axis aligned to the radius of the dish and the center of their head $7 \mathrm{~mm}$ from the wall. Once the agarose had set, it was covered with aquarium water and an ophthalmic scalpel was used to dissect sections away so as to permit free movement of the eyes and the tail. The only part of the larvae that remained embedded in agarose was from the otic vesicle to the posterior end of the swim bladder. Larvae were tested at 7 or 8 dpf. A diffusive filter 3026 (Rosco, Inc., Hollywood, CA) was affixed to the wall of the Petri dish to produce a $>180^{\circ}$ projection screen directly in front of the fish. The dish was then placed on a stage within a light-tight box, and was illuminated from below with dim white light (12 lux), as well as $850 \mathrm{~nm}$ infrared light, provided by LEDs. Larvae were imaged from above at $100 \mathrm{~Hz}$ using a high-speed camera (Pike F-032B, Allied Vision Technologies), fitted with a machine vision lens (Kowa LM35JCM), a $15 \mathrm{~mm}$ extension tube and an infrared bandpass filter (BP850, Midwest Optical Systems).

Experiments were controlled using software custom-written in LabView (National Instruments, Austin, TX). The orientation of the left and right eyes in the horizontal plane was determined by fitting an ellipse to each eye and recording the angle between the long axis of the ellipse and a line parallel to the midline of the larva. Positive angles were measured clockwise from the midline to the long axis of the ellipse and negative angles counterclockwise. For both eyes, increases in eye angle indicate rightward rotation and decreases, leftward rotation. The tail was detected as $10 \mathrm{x}-\mathrm{y}$ co-ordinates.

Visual stimuli were designed using custom software written in MatLaв (The MathWorks Inc.) using the Psychophysics toolbox (Brainard, 1997; Pelli, 1997) and were presented using a laser pico-projector (SHOWWX, Microvision) positioned $12 \mathrm{~cm}$ from the diffusive screen. Visual cues were bright white spots $\sim 1 \mathrm{~mm}$ in diameter, presented at maximum contrast. For "leftward" and "rightward" moving stimuli, spots were presented for $3 \mathrm{~s}$, appearing directly in front of the fish and moving around the screen at $\sim 30^{\circ}$ s to a final location $94^{\circ}$ to the left or right of the extended midsaggital plane of the larva. "Center" stimuli oscillated back and forth for $5 \mathrm{~s}$ in a sinusoidal manner, $\pm 25^{\circ}$ either side of the extended midsaggital plane of the larva.

Experiments consisted of approximately 100 trials of $20 \mathrm{~s}$ each. In two-thirds of trials, no stimulus was presented, allowing us to evaluate the frequency of spontaneous eye convergence. In every third trial a stimulus was shown, starting at the $5 \mathrm{~s}$ time-point. An equal number of each stimulus type was presented, in random sequence.

\section{DATA ANALYSIS}

All data was analyzed using software custom-written in MatLAB. For experiments with restrained larvae, we detected saccades by convolving the vector describing angular eye position over time with a step filter, and detected peaks exceeding a manually defined threshold. Eye movements were classified as convergences when both eyes made a nasally directed saccade within $150 \mathrm{~ms}$ of one another. We define vergence angle as the difference between left and right eye position; nasal rotation of either eye causes an increase in vergence angle.

To evaluate tail movements, we computed the 8 angles between the 9 line-segments interconnecting the $10 \mathrm{x}$-y points that were used to define the tail at each time-point. Rightward bending of the tail is represented by positive angles and leftward bending by negative angles. We reported the proportion of total tail curvature localized to the final $22 \%$ of tail length by dividing the sum of the final two tail angles (contributed by the final 2 line-segments) by the sum of all 8 angles (contributed by all 9 line-segments).

Note that for the example trials shown in Figures 3 and 5, data traces describing eye position and cumulative tail angle have been reflected along their $\mathrm{x}$-axes to provide a conventional representation of eye convergence (the left and right eye position traces approach one another during a convergence).

All summary data are presented as mean \pm s.e.m. unless stated otherwise. The statistical tests used are stated in the Results and significance was considered at a 95\% confidence limit.

\section{ACKNOWLEDGMENTS}

The authors thank Ruben Portugues and David Schoppik for helpful discussions and critical reading of the manuscript. Isaac $\mathrm{H}$. Bianco is supported by a Sir Henry Wellcome Postdoctoral Fellowship from the Wellcome Trust. Research was supported by NIH grants 1R01DA030304-01 and 1RC2NS069407-01 awarded to Florian Engert.

\section{SUPPLEMENTARY MATERIAL}

The Movies S1, S2, and S3 for this article can be found online at http://www.frontiersin.org/Systems_Neuroscience/10.3389/fnsys. 2011.00101/abstract

Movie S1 | Hunting routine of a freely swimming zebrafish larva preying on a paramecium. Movie was acquired at $80 \mathrm{~Hz}$ and is played back at $5 \mathrm{~Hz}$. Notice that the zebrafish used multiple J-turns to reorient toward the paramecium, and that eye convergence persists for the duration of the hunting routine, until just after the larva strikes at its prey. 
Movie S2 | Restrained larva responding to a leftward-moving spot. Movie was acquired at $100 \mathrm{~Hz}$ and is played back at $5 \mathrm{~Hz}$. The zebrafish responds with eye convergence and leftward J-bends.

\section{REFERENCES}

Beck, J. C., Gilland, E., Tank, D. W., and Baker, R. (2004). Quantifying the ontogeny of optokinetic and vestibuloocular behaviors in zebrafish, medaka, and goldfish. J. Neurophysiol. 92, 3546-3561.

Borla, M. A., Palecek, B., Budick, S., and O'Malley, D. M. (2002). Prey capture by larval zebrafish: evidence for fine axial motor control. Brain Behav. Evol. 60, 207-229.

Brainard, D. H. (1997). The psychophysics toolbox. Spat. Vis. 10, 433-436.

Burgess, H. A., and Granato, M. (2007). Sensorimotor gating in larval zebrafish. J. Neurosci. 27, 4984-4994.

Burgess, H. A., Schoch, H., and Granato, M. (2010). Distinct retinal pathways drive spatial orientation behaviors in zebrafish navigation. Curr. Biol. 20, 381-386.

Creton, R. (2009). Automated analysis of behavior in zebrafish larvae. Behav. Brain Res. 203, 127-136.

Douglass, A. D., Kraves, S., Deisseroth, K., Schier, A. F., and Engert, F. (2008). Escape behavior elicited by single, channelrhodopsin-2-evoked spikes in zebrafish somatosensory neurons. Curr. Biol. 18, 1133-1137.

Easter, S. S. Jr., and Nicola, G. N. (1996). The development of vision in the zebrafish (Danio rerio). Dev. Biol. 180, 646-663.

Easter, S. S. Jr., and Nicola, G. N. (1997). The development of eye movements in the zebrafish (Danio rerio). Dev. Psychobiol. 31, 267-276.

Ewert, J. P., Buxbaum-Conradi, H., Dreisvogt, F., Glagow, M., Merkel-Harff, C., Röttgen, A., Schürg-Pfeiffer, E., and Schwippert, W. W. (2001). Neural modulation of visuomotor functions underlying prey-catching behaviour in anurans: perception, attention, motor performance, learning. Comp. Biochem. Physiol. Part A Mol. Integr. Physiol. 128, 417-461.
Gahtan, E., Tanger, P., and Baier, H. (2005). Visual prey capture in larval zebrafish is controlled by identified reticulospinal neurons downstream of the tectum. J. Neurosci. 25, 9294-9303.

Higashijima, S.-I., Masino, M. A., Mandel, G., and Fetcho, J. R. (2003) Imaging neuronal activity during zebrafish behavior with a genetically encoded calcium indicator. $J$. Neurophysiol. 90, 3986-3997.

Hunter, J. (1972). Swimming and feeding behavior of larval anchovy engraulis-mordax. Fish. Bull. 70, 821

Janovjak, H., Szobota, S., Wyart, C., Trauner, D., and Isacoff, E. Y. (2010). A light-gated, potassium-selective glutamate receptor for the optical inhibition of neuronal firing. Nat. Neurosci. 13, 1027-1032.

Kimmel, C. B., Patterson, J., and Kimmel, R. O. (1974). The development and behavioral characteristics of the startle response in the zebra fish. Dev. Psychobiol. 7, 47-60.

Liu, K. S., and Fetcho, J. R. (1999). Laser ablations reveal functional relationships of segmental hindbrain neurons in zebrafish. Neuron 23, 325-335.

Luque, M. A., Pérez-Pérez, M. P., Herrero, L., Waitzman, D. M., and Torres, B. (2006). Eye movements evoked by electrical microstimulation of the mesencephalic reticular formation in goldfish. Neuroscience 137, 1051-1073.

MacKenzie, B., and Kiorboe, T. (2000). Larval fish feeding and turbulence: a case for the downside. Limnol. Oceanogr. 45, 1-10.

Mays, L. E., Porter, J. D., Gamlin, P. D., and Tello, C. A. (1986). Neural control of vergence eye movements: neurons encoding vergence velocity. J. Neurophysiol. 56, 1007-1021.

McElligott, M. B., and O'Malley, D. M. (2005). Prey tracking by larval zebrafish: axial kinematics and visual control. Brain Behav. Evol. 66, 177-196.

Movie S3 | Restrained larva responding to a rightward-moving spot. Movie was acquired at $100 \mathrm{~Hz}$ and is played back at $5 \mathrm{~Hz}$. This is the same fish as in Movie S2. The zebrafish responds with eye convergence and rightward J-bends.

McLean, D. L., Fan, J., Higashijima, S.-I., Hale, M. E., and Fetcho, J. R. (2007) A topographic map of recruitment in spinal cord. Nature 446 71-75.

McLean, D. L., and Fetcho, J. R. (2011) Movement, technology and discovery in the zebrafish. Curr. Opin. Neurobiol. 21, 110-115.

Munk, P. (1995). Foraging behavior of larval cod (Gadus morhua) influenced by prey density and hunger. Mar. Biol. 122, 205-212.

Muto, A., Ohkura, M., Kotani, T., Higashijima, S.-I., Nakai, J., and Kawakami, K. (2011). Genetic visualization with an improved gcamp calcium indicator reveals spatiotemporal activation of the spinal motor neurons in zebrafish. Proc. Natl. Acad. Sci. U.S.A. 108, 5425-5430.

Orger, M. B., Gahtan, E., Muto, A. Page-McCaw, P., Smear, M. C., and Baier, H. (2004). Behavioral screening assays in zebrafish. Methods Cel Biol. 77, 53-68.

Orger, M. B., Kampff, A. R., Severi, K. E., Bollmann, J. H., and Engert, F. (2008). Control of visually guided behavior by distinct populations of spinal projection neurons. $\mathrm{Nat}$. Neurosci. 11, 327-333.

Pelkowski, S. D., Kapoor, M., Richendrfer, H. A., Wang, X., Colwill R. M., and Creton, R. (2011). A novel high-throughput imaging system for automated analyses of avoidance behavior in zebrafish larvae. Behav. Brain Res. 223, 135-144.

Pelli, D. G. (1997). The videotoolbox software for visual psychophysics: transforming numbers into movies. Spat. Vis. 10, 437-442.

Portugues, R., and Engert, F. (2009). The neural basis of visual behaviors in the larval zebrafish. Curr. Opin. Neurobiol. 19, 644-647.

Prete, F. R., Wells, H., Wells, P. H., and Hurd, L. E. (eds). (1999). The Praying Mantids. Baltimore, MA: The Johns Hopkins University Press.
Salas, C., Herrero, L., Rodriguez, F., and Torres, B. (1997). Tectal codification of eye movements in goldfish studied by electrical microstimulation. $\mathrm{f}$. Neuroscience 78, 271-288.

Satou, C., Kimura, Y., Kohashi, T., Horikawa, K., Takeda, H., Oda, Y., and Higashijima, S.-I. (2009). Functional role of a specialized class of spinal commissural inhibitory neurons during fast escapes in zebrafish. J. Neurosci. 29, 6780-6793.

Schoonheim, P. J., Arrenberg, A. B., Del Bene, F., and Baier, H. (2010). Optogenetic localization and genetic perturbation of saccade-generating neurons in zebrafish. J. Neurosci. 30, 7111-7120.

Vollset, K. W., Folkvord, A., and Browman, H. I. (2011). Foraging behaviour of larval cod (Gadus morhua) at low light intensities. Mar. Biol. 158, 1125-1133.

Wyart, C., Del Bene, F., Warp, E., Scott, E. K., Trauner, D., Baier, H., and Isacoff, E. Y. (2009). Optogenetic dissection of a behavioural module in the vertebrate spinal cord. Nature 461, 407-410.

Conflict of Interest Statement: The authors declare that the research was conducted in the absence of any commercial or financial relationships that could be construed as a potential conflict of interest.

Received: 09 November 2011; accepted: 29 November 2011; published online: 16 December 2011.

Citation: Bianco IH, Kampff $A R$ and Engert F (2011) Prey capture behavior evoked by simple visual stimuli in larval zebrafish. Front. Syst. Neurosci. 5:101. doi: 10.3389/fnsys.2011.00101

Copyright (c) 2011 Bianco, Kampff and Engert. This is an open-access article distributed under the terms of the Creative Commons Attribution Non Commercial License, which permits non-commercial use, distribution, and reproduction in other forums, provided the original authors and source are credited. 